\section{Superior colliculus saccade motor bursts do not dictate movement kinematics}

\author{
Ziad M. Hafed ${ }^{1,2}$ \\ ${ }^{1}$ Werner Reichardt Centre for Integrative Neuroscience, Tübingen University, \\ Tübingen, Germany \\ ${ }^{2}$ Hertie Institute for Clinical Brain Research, Tübingen University, \\ Tübingen, Germany
}

\section{Correspondence}

\author{
Werner Reichardt Centre for Integrative Neuroscience \\ Tübingen University \\ Otfried-Müller Str. 25 \\ Tübingen, 72076, Germany \\ Tel: +4970712988819 \\ ziad.m.hafed@cin.uni-tuebingen.de
}

\title{
Introduction
}

The superior colliculus (SC) plays an important role in saccade generation, as evidenced by the ease with which low-current electrical microstimulation of SC neurons evokes saccades (Katnani \& Gandhi, 2012; Robinson, 1972). Anatomically, SC neurons are organized to form a spatial code of eye movement displacement vectors (Cynader \& Berman, 1972; Lee, Rohrer, \& Sparks, 1988; Robinson, 1972), such that the location of an active neuron in the SC defines the amplitude and direction of a desired saccade. Robustness and accuracy of saccade vector representation are ensured through population coding (Lee et al., 1988; Massot, 
Jagadisan, \& Gandhi, 2019; Munoz \& Wurtz, 1995b), with the aggregate activity of a large number of simultaneously active neurons defining a given movement's metrics.

The SC spatial code necessarily entails a temporal synchrony of SC activity at the time of saccades. Indeed, saccade-related neurons show a highly characteristic temporal evolution of spiking (Jagadisan \& Gandhi, 2019; Munoz \& Wurtz, 1995a; Schiller \& Koerner, 1971; Sparks, Holland, \& Guthrie, 1976; Wurtz \& Goldberg, 1971), dominated by a burst tightly locked to movement onset. Interestingly, the strength of such a burst can vary, suggesting that SC neurons may encode additional properties beyond the saccadic displacement vector represented by the spatial code. For example, blink-perturbed saccades can have weaker, but prolonged, bursts (Goossens \& Van Opstal, 2000, 2012). Moreover, burst evolution during a saccade may be related to the remaining motor error of an ongoing eye movement (Waitzman, Ma, Optican, \& Wurtz, 1988, 1991) (i.e. how much more the eye needs to keep moving), or it may be related to the speed profile of the ensuing saccade (Smalianchuk, Jagadisan, \& Gandhi, 2018). Additionally, saccade-related burst strength can be modulated by audio-visual sensory combinations (Frens \& Van Opstal, 1998). Thus, there is an SC temporal code for saccades, the role of which is less well understood than that of the spatial code.

The most recent SC models posit an important role for the temporal code in dictating millisecond-by-millisecond movement trajectory (Goossens \& van Opstal, 2012; Smalianchuk et al., 2018; van Opstal \& Goossens, 2008). In these models, the locus of an active neuron (i.e. the spatial code) defines how each individual spike in a motor burst moves the eye; parameters like eye speed or time to movement end would reflect the strength of (i.e. number of spikes in) the motor burst (i.e. the temporal code). While appealing in their combination of both spatial and temporal codes for movement specification, these models suggest a very tight relationship between saccade-related burst strength and movement kinematics. However, this may not necessarily always be the case. For example, we recently explored a situation in which saccade kinematics are altered by a simultaneity condition between a motor burst somewhere on the SC map and an irrelevant visual burst somewhere else (Buonocore et al., 2017; Buonocore, McIntosh, \& Melcher, 2016). When we recorded at both the motor and visual burst locations (Buonocore et al., 2021), we found a lawful relationship between the saccade metric changes and the number of additional spikes injected by the visual burst (consistent with the spatial code); however, critically, the simultaneous motor burst was minimally affected (Buonocore et al., 2021). Thus, the temporal code was essentially unaltered even though the movements themselves were. This, along with other evidence (e.g. (Peel, Dash, Lomber, \& Corneil, 2020), motivates investigating whether saccade kinematics are indeed dictated by the SC temporal code or not.

I approached this question by exploiting a large asymmetry in how the SC represents the upper versus lower visual fields in its visual sensitivity (Hafed \& Chen, 2016). If such an asymmetry still holds, but now for saccade-related motor burst strength, then there should be (according to current models of the temporal code) systematic differences in the saccades' kinematics. I confirmed a neural asymmetry in SC motor burst strengths, but found no concomitant kinematic differences between saccades towards the upper and lower visual fields. My observations highlight the need to explore other potential functional roles for the saccade-related SC temporal code. 


\section{Results}

I identified a dissociation between SC motor burst strengths and their associated eye movements' kinematics. Specifically, I explored how SC motor burst strength might differ as a function of visual field location. When we recently described an asymmetry in how the SC represents the upper and lower visual fields (Hafed \& Chen, 2016), we found that SC visual response properties were different across the fields. We also briefly mentioned that the strength of saccade-related motor bursts may also be asymmetric (Hafed \& Chen, 2016). Here, I investigated the robustness of this saccade-related neural asymmetry in more detail, and I then asked whether it predicted an asymmetry in saccade kinematics between movements towards the upper and lower visual fields.

\section{Difference in superior colliculus motor burst strengths for saccades towards the upper versus lower visual fields}

Visual sensitivity is significantly stronger in SC neurons representing the upper visual field (Hafed \& Chen, 2016). That is, if a neuron's visual response field has a preferred (hotspot) location above the retinotopic horizontal meridian and we present a stimulus at this location, then the neuron's response is stronger than that of a neuron with a lower visual field hotspot location (and a stimulus presented at its preferred location). Curiously, in our earlier study (Hafed \& Chen, 2016), we noticed that saccade-related motor bursts showed the opposite asymmetry: saccade-related motor bursts (for preferred hotspot locations) were stronger for neurons representing saccades towards the lower visual field than for neurons representing saccades towards the upper visual field. However, in that study (Hafed \& Chen, 2016), we did not control for the depths of the recorded neurons from the SC surface when we analyzed the neurons' motor bursts. Since the strength of SC motor bursts can vary substantially with depth from the SC surface (Massot et al., 2019), here, I wanted to first confirm whether the asymmetry alluded to above (Hafed \& Chen, 2016) was still present when carefully controlling for neuron depth (Methods). If this was the case, I could then ask whether saccade kinematics were systematically different or not.

I re-analyzed the neural database of (Hafed \& Chen, 2016) by first matching the depths of neurons from the SC surface between the upper and lower visual fields (Methods). For each neuron in this database (here referred to as dataset 1), I identified whether the neuron was saccade-related or not (Hafed \& Chen, 2016). I then classified the saccade-related neurons as having a movement-related preferred response field location (or hotspot location) in the upper or lower visual field. Preference was defined as the location for which saccades were associated with the highest firing rates, similarly to how visual preference was defined as the location for which visual stimuli evoked the strongest visual bursts (Hafed \& Chen, 2016). Finally, I picked a range of neural depths from the SC surface that was overlapping between the upper and lower visual field neurons. This final step was the critical step for the present analysis, and it resulted in me having a total of 167 SC neurons with depths from the SC surface between 600 and $1850 \mu \mathrm{m}$ (Methods). The distribution of these depths is represented in Fig. 1A, where each neuron's depth is plotted against a measure of whether the neurons' preferred movement-related response field location was above or below the horizontal meridian (the $\mathrm{x}$-axis shows the direction of the preferred location from the horizontal meridian; positive means above the meridian, and negative means below). As can 
be seen, I found saccade-related SC activity at a range of depths from the SC surface that was consistent with prior observations (Massot et al., 2019; Mohler \& Wurtz, 1976; Munoz \& Wurtz, 1995a; Schiller \& Koerner, 1971; Sommer \& Wurtz, 2004; Sparks et al., 1976; Wurtz \& Goldberg, 1971). Critically, the neural depths were overlapping between the upper and lower visual field neurons (Fig. 1A); note (as an aside) that upper visual field directions were compressed relative to lower visual field directions, which is consistent with the idea of upper visual field neural tissue magnification in the SC (Hafed \& Chen, 2016). Therefore, I was now in a position to check whether an asymmetry of saccade-related burst strengths alluded to earlier (Hafed \& Chen, 2016) still held after controlling for neuron depth.

152

153

154

155
A

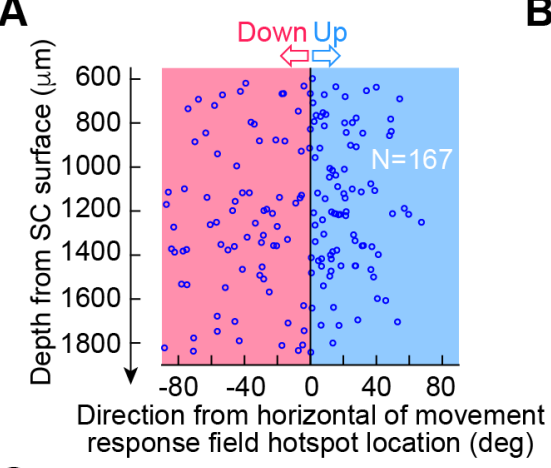

C

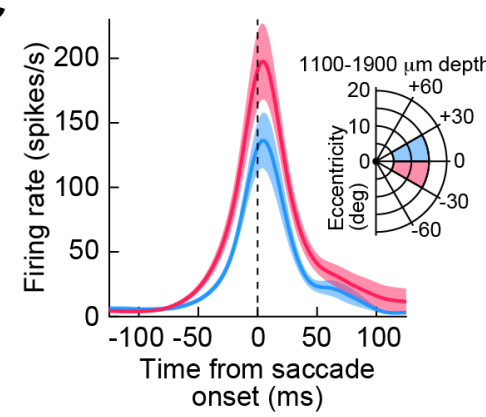

B
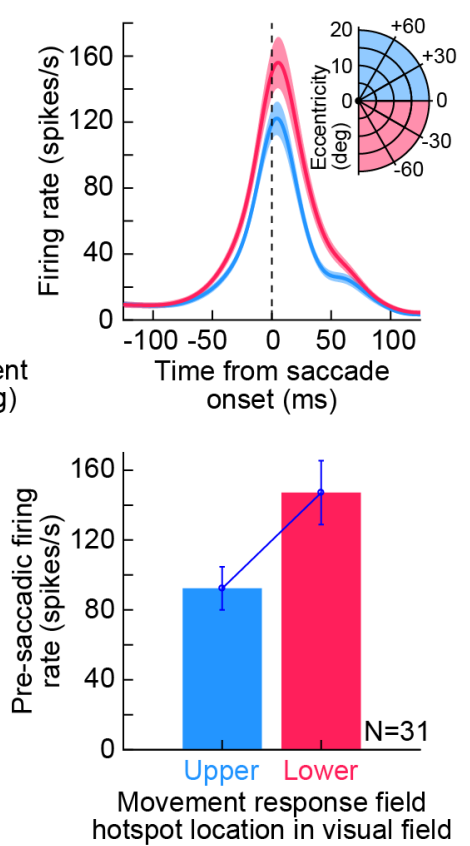

Figure 1 Superior colliculus (SC) saccade-related motor bursts are stronger for downward saccades than for upward saccades, even when controlling for depth from the SC surface. (A) I picked neurons matched for depth from the SC surface (between 600 and $1850 \mu \mathrm{m}$ ) but having movement field hotspot locations in either the upper (light blue) or lower (light red) visual fields (i.e. positive or negative directions from the horizontal meridian, respectively). Note that the upper visual field neurons appear compressed along the direction dimension (i.e. in visual coordinates), likely due to upper visual field neural tissue magnification (Hafed \& Chen, 2016). Such magnification is similar, in principle, to foveal magnification of SC neural tissue (Chen, Hoffmann, Distler, \& Hafed, 2019). (B) For the neurons in A, I plotted peri-saccadic firing rates for saccades towards each neuron's preferred movement field location (Hafed \& Chen, 2016). Saccade-related bursts were stronger for lower visual field than upper visual field neurons. (C) To better constrain errors in depth estimates from the SC surface (due to surface curvature), I further restricted the choice of neurons to those primarily near the horizontal meridian and at an eccentricity range associated with quasi-constant tissue curvature between upper and lower visual field locations (Chen et al., 2019); the ranges of amplitudes, directions, and depths are shown in the inset. The motor bursts of the resulting 31 neurons were still stronger for lower visual field than upper visual field neurons (left panel). The right panel shows average firing rate in the final $50 \mathrm{~ms}$ before saccade onset; lower visual field neurons exhibited stronger activity than upper visual field neurons ( $p=0.0162$; t-test; t-statistic: -2.5524 ; df: 29). Error bars in all panels denote s.e.m. 
Having established that I now had a neural database with matched depths from the SC surface, I proceeded to comparing motor burst strengths between the upper and lower visual field neurons. I plotted the peri-saccadic firing rates of the neurons of Fig. 1A. I specifically picked, for each neuron, the preferred saccades of the neuron and plotted its firing rate for these movements, as we did previously (Hafed \& Chen, 2016). I then averaged across all neurons (Fig. 1B). There was indeed an asymmetry in SC motor burst strengths, such that neurons representing the lower visual field had significantly stronger motor bursts than neurons representing the upper visual field (Fig. 1B). To statistically assess the difference in burst strengths after matching for neural depths from the SC surface, I measured the average firing rate in the final 50 ms before saccade onset for each neuron's preferred saccades (Hafed \& Chen, 2016) (Methods). I then compared the population of measurements for the upper and lower visual field neurons of Fig. 1A using a t-test. Across neurons, average firing rate for the upper visual field neurons was $99 \mathrm{spikes} / \mathrm{s}$, and it was $121 \mathrm{spikes} / \mathrm{s}$ for the lower visual field neurons. This difference was statistically significant ( $p=0.039$; t-statistic: -2.0844 ; df: 134). Therefore, even after controlling for the depths of neurons from the SC surface, I confirmed a potential asymmetry in saccade-related burst strength between upper and lower visual field saccades (Hafed \& Chen, 2016).

A potential concern related to the above interpretation might be the curvature associated with the SC's 3-dimensional shape. Since all electrode paths were constant and defined by the recording chamber's orientation (Methods), it could still be possible that lateral recording sites (representing the lower visual field) could have had systematically different depths from the SC surface than medial recording sites (representing the upper visual field), by virtue of the different SC surface curvature at the two groups of sites. I therefore decided to analyze a stricter grouping of SC neurons. I picked a smaller range of eccentricities (5-15 $\mathrm{deg}$ ), directions from the horizontal meridian (<30 deg), and depths from the SC surface (1100-1900 $\mu \mathrm{m})$ for comparing upper and lower visual field neurons' motor bursts. Our prior work on SC surface topography and 3-dimensional anatomical shape (Chen et al., 2019) suggested that this range of selection should reduce potential systematic differences in estimates of depths from the SC surface between the upper and lower visual field groups of neurons. I found 31 neurons (20 upper visual field and 11 lower visual field) satisfying the above strict criteria. When I analyzed their saccade-related firing rates, I still found a similar asymmetry between upper and lower visual field locations (Fig. 1C). Therefore, it is likely given both analyses in Fig. 1 that there is indeed a systematic asymmetry in SC motor burst strength between saccades towards the upper and lower visual fields. I was now in a position to ask whether such an asymmetry was reflected in saccade kinematics, as might be predicted from some recent models of the SC temporal code (Goossens \& van Opstal, 2012; Smalianchuk et al., 2018; Waitzman et al., 1991).

\section{Similarity of movement kinematics for saccades towards the upper and lower visual fields}

I collected saccades from both monkeys from the same task that was used to analyze the peri-saccadic SC firing rates above (Methods). To compare size- and direction-matched movements, I picked, in each monkey, 5 saccade sizes $(3,5,7,10$, and 13 deg radial amplitude), and two example directions from the horizontal meridian (+45 and -45 deg; i.e. oblique saccades; note that I also made similar observations for example directions that were nearer to or farther away from the horizontal meridian than $+/-45 \mathrm{deg}$ ). For each of 
the saccade sizes, I picked movements landing within a radius of $0.5,0.8,1,2$, and 3 deg, respectively for the increasing saccade amplitude categories listed above. Therefore, I ensured that the movement endpoints were matched for landing accuracy. Example such movements are shown in Fig. 2A. In this figure, I only plotted rightward movements in monkey $\mathrm{N}$ and leftward movements in monkey $\mathrm{P}$, for simplicity, but Fig. 2B shows both rightward and leftward saccades in each of the two monkeys. As can be seen from Fig. 2A, there was no clear difference in the trajectories of upward (light blue) versus downward (light red) oblique saccades, despite the significant SC neural asymmetry in Fig. 1. In fact, the pink upward traces in Fig. $2 \mathrm{~A}$ are the same as the light red downward traces in the figure, but now reflected across the horizontal meridian for easier comparison to the upward saccades shown in light blue. These pink traces clearly overlapped strongly in their temporal evolution with the upward saccades.

A

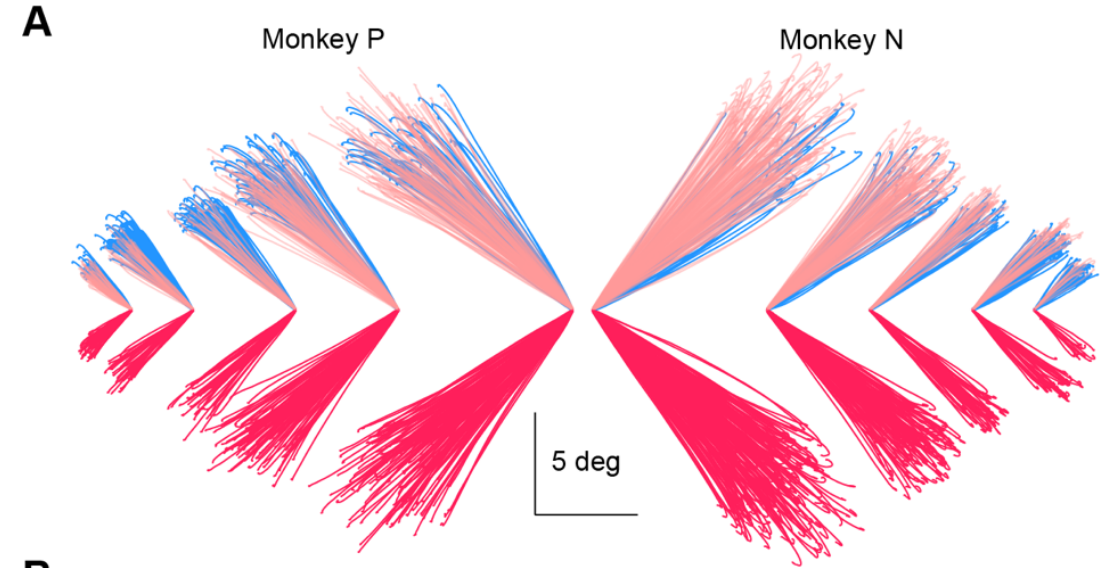

B
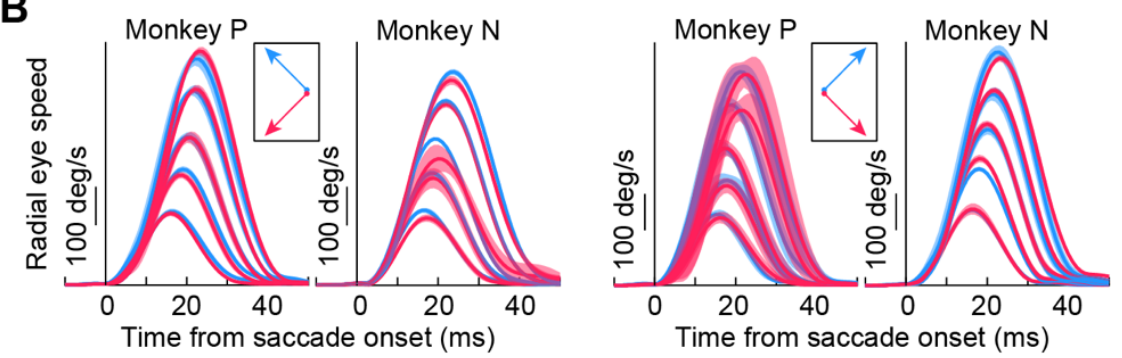

Figure 2 Upward and downward oblique saccades from the same sessions as the neural recordings in Fig. 1 exhibited similar kinematic properties, despite the neural asymmetry of Fig. 1. (A) Oblique saccades of different directions and amplitudes in both monkeys (rightward for $\mathrm{N}$ and leftward for P). Each line plots the horizontal and vertical displacement of eye position for a given saccade having a +45 deg (light blue) or -45 deg (light red) direction from the horizontal meridian. Saccades of similar sizes are grouped together (to start from the same origin) and displaced horizontally in the figure from saccades of different size ranges (the scale bars apply to all sizes). The pink traces overlaying the light blue traces are the same as the light red traces of the lower visual field saccades, but now reflected along the vertical dimension for better comparison to the upper visual field saccades. The trajectories of the saccades were largely similar regardless of direction from the horizontal meridian, despite the asymmetry in motor bursts in Fig. 1. (B) For each monkey, I plotted radial eye speed as a function of time from saccade onset for leftward (left pair of plots) or rightward (right pair of plots) saccades. In each case, I separated upward and downward movements by color (as in A). The different saccade sizes in $\mathbf{A}$ are reflected in the different peak velocities (Bahill, Clark, \& Stark, 1975; Zuber, Stark, \& Cook, 1965). The kinematic time courses of saccade acceleration, peak velocity, and deceleration were largely similar for upward and downward saccades, despite the asymmetry of SC motor bursts in Fig. 1. Error bars denote $95 \%$ confidence intervals. The numbers of trials per condition in monkey $\mathrm{N}$ ranged from 7 to 362 (mean 121.2), and the numbers of trials per condition in monkey $P$ ranged from 5 to 252 (mean 72.25). 
Across the population of measurements from the above saccades, I plotted the radial eye speed as a function of saccade amplitude and direction (Fig. 2B). This kind of plot summarizes the kinematics of the eye movements (Bahill et al., 1975; Zuber et al., 1965). For each saccade size and right/left direction in each monkey, I plotted the radial eye speed for either upward (light blue) or downward (light red) oblique saccades (error bars denote 95\% confidence intervals). There were no systematic differences in the saccadic profiles of the two groups of movements (across all sizes tested), despite the systematically stronger SC motor bursts for downward saccades seen in Fig. 1 (compare light blue and light red profiles for each saccade size). For example, stronger motor bursts in Fig. 1 could have predicted systematically higher peak speeds for the saccades directed towards the lower visual field. This was clearly not the case (Fig. 2B). In fact, lower visual field neurons possess larger movement fields than upper visual field neurons (Hafed \& Chen, 2016), which should further increase the number of "active" spikes during saccade-related bursting for saccades towards the lower visual field; nonetheless, the kinematics of the movements were largely the same as those of upper visual field saccades (Fig. 2). Therefore, there is a dissociation between SC saccade-related motor burst strength (Fig. 1) and saccade kinematics (Fig. 2).

\section{Similarity of upper and lower visual field saccade kinematics for a variety of behavioral contexts}

I next turned to another, larger database of saccades for analyzing kinematics in more detail (dataset 2; Methods). In this case, I used: 1) visually-guided, 2) delayed, visually-guided, and 3) memory-guided saccades of different sizes and directions, with the sizes ranging from those associated with fixational microsaccades (approximately 0.1-0.2 deg) to approximately 15-20 deg. Aspects of these movements were analyzed previously for other purposes than movement kinematics (Hafed \& Goffart, 2020; Willeke et al., 2019). Here, I wanted to confirm that the results of Fig. 2 still held for a larger range of movement amplitudes and directions, and also under different behavioral contexts. In other words, I analyzed the movements' kinematic properties in data set 2, properties which were not analyzed in the prior publications. Moreover, dataset 2 allowed me to include data from a third monkey, $M$, when assessing potential differences (or lack thereof) in saccade kinematics between the upper and lower visual fields.

In our recent work with this dataset (Hafed \& Goffart, 2020), we reported that saccadic reaction times were systematically shorter for upper visual field target locations when compared to lower visual field target locations, consistent with the asymmetry of SC visual neural sensitivity (Hafed \& Chen, 2016), and also consistent with other behavioral evidence (Greene, Brown, \& Dauphin; Greenwood, Szinte, Sayim, \& Cavanagh, 2017; Schlykowa, Hoffmann, Bremmer, Thiele, \& Ehrenstein, 1996; Zhou \& King, 2002). For example, in Fig. 3A, I plotted example oblique saccades from monkey $\mathrm{N}$ from this new dataset (but in a format similar to that used in plotting the data of Fig. 2A). In Fig. 3B (left), I plotted the absolute value of vertical eye position from the saccades shown in Fig. $3 \mathrm{~A}$ (to facilitate comparing the upward and downward movements). Here, I temporally aligned the movements to the time of the go signal for triggering the saccades (the peripheral targets were continuously visible). Even though the saccade trajectories looked similar in Fig. 3A (save for the upward and downward distinction), the reaction times of the movements were markedly different (Fig. $3 \mathrm{~B}$, left). Saccades towards the upper visual field were triggered significantly earlier than 
saccades towards the lower visual field (Hafed \& Goffart, 2020). I then replotted the same saccades, but this time by aligning them to the time of peak radial eye speed during the movements (Fig. 3B, right). The movement trajectories (i.e. kinematics) were largely overlapping, with similar acceleration and deceleration profiles.

A

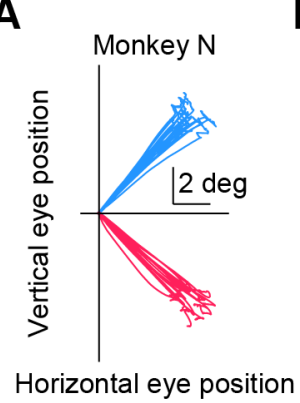

B

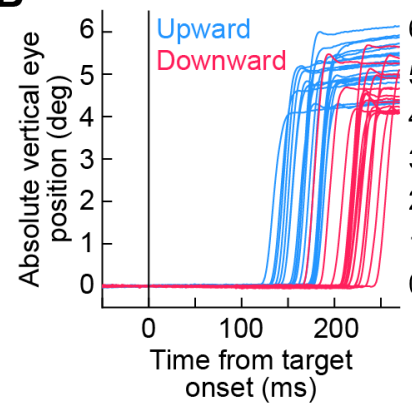

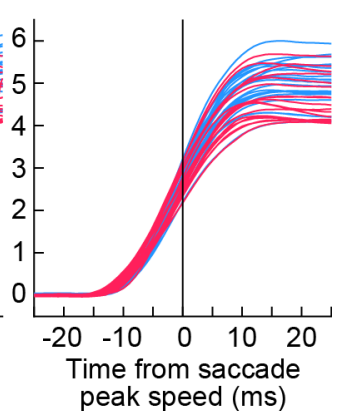

Figure 3 Upward and downward saccades exhibit strong differences in reaction times, but not kinematics. (A) From the second dataset, I plotted, by way of example, similarly-sized oblique saccades from one monkey, and I separated them as being either upward or downward (as in Fig. 2A). (B) Relative to stimulus onset (left panel), the saccades were very different from each other in terms of their reaction times, as we characterized in detail earlier (Hafed \& Goffart, 2020); saccades towards the upper visual field (light blue) had significantly shorter reaction times than saccades towards the lower visual field (light red). However, kinematically, the saccades were very similar when aligned to the time of peak intra-saccadic eye speed (right panel). In both panels, I plotted the absolute value of vertical eye position displacement for each saccade (for better comparison of the upward and downward movements). Figures 4, 5 summarize the saccade kinematic results that I obtained for a much larger number of movements, and for two monkeys. $\mathrm{N}=21$ upward saccades and $\mathrm{N}=18$ downward saccades in this figure.

To summarize this kinematic similarity across all saccades in this new dataset, I generated plots of peak eye speed as a function of saccade amplitude for each monkey (Bahill et al., 1975; Zuber et al., 1965). In the first row of Fig. 4, these plots were made for the immediate, visually-guided saccade task, in which the fixation spot was extinguished at the same time as the appearance of the eccentric stimulus (Methods). For both monkeys $\mathrm{N}$ and $\mathrm{M}$, there was very minimal difference in the main sequence relationship between saccades towards the upper (light blue) or lower (light red) visual fields, and any difference was certainly much smaller than the neural effects in Fig. 1. In fact, the insets in the first row of Fig. 4 show the reaction time results for the very same saccades, which are replicated from our recent work (Hafed \& Goffart, 2020) for clarity. Despite a large effect of the visual field location on the movements' reaction times (also seen in Fig. 3B, left), there was minimal difference in saccade kinematics. This is again supportive of a dissociation between saccade-related SC motor burst strengths (Fig. 1) and movement kinematics (Figs. 2, 3).

With an even larger database of visually-guided movements in this dataset, now from the delayed, visually-guided saccade task, the same conclusion could be reached: the middle row of Fig. 4 shows virtually no difference in the saccade kinematics between upward and downward visually-guided saccades, despite a clear effect size for SC motor-related neural responses in Fig. 1 and (Hafed \& Chen, 2016). The peak speeds in this row were also consistent with the peak speeds in the first row of Fig. 4 obtained with the immediate, visually-guided saccade task, as might be expected given the presence of a visual target for the saccades in both tasks. 

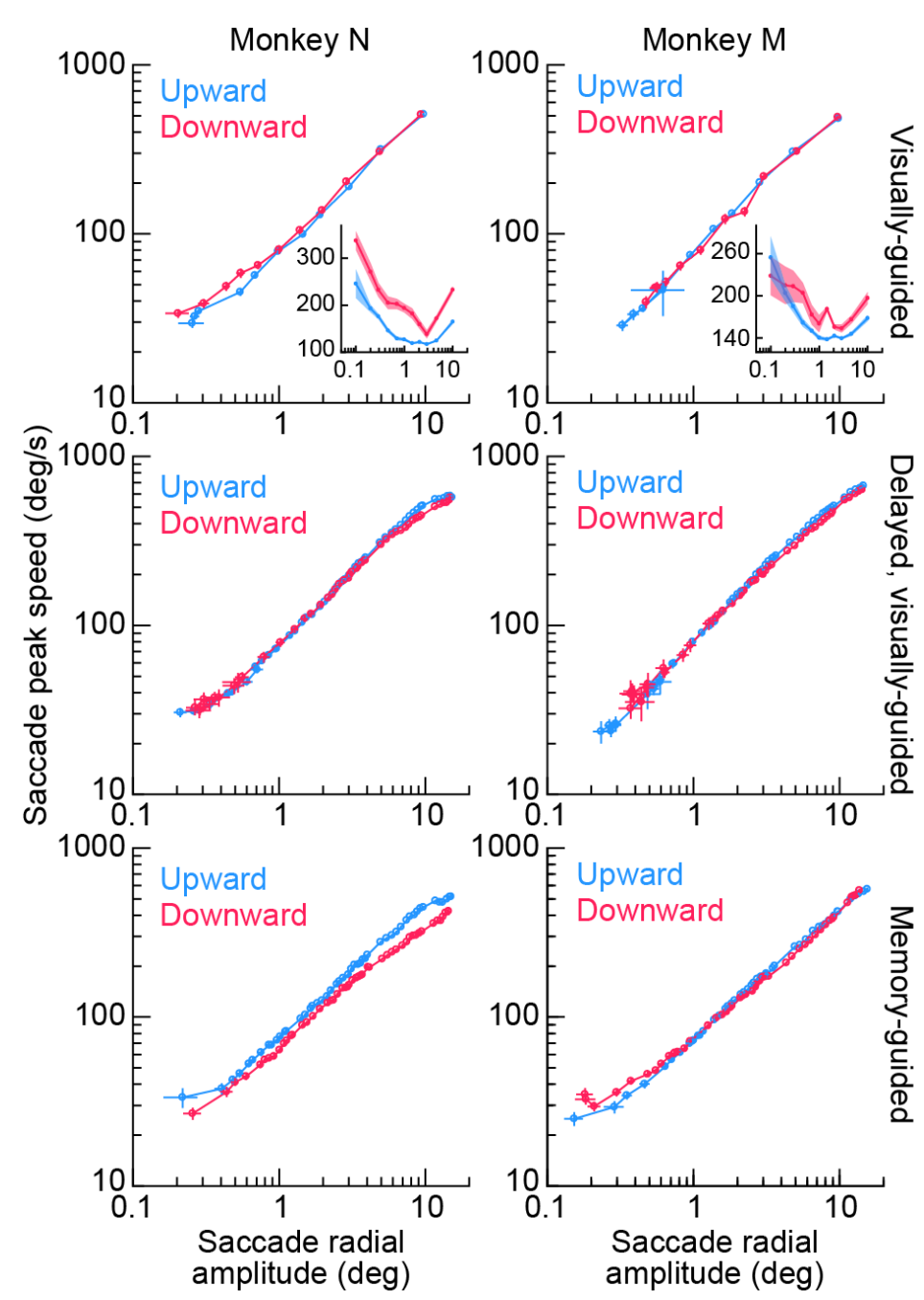

Figure 4 The main sequence relationship between peak eye speed and saccade amplitude does not depend on whether saccades are upward or downward for a variety of behavioral task contexts. In each monkey (left and right columns), I plotted the main sequence from dataset 2 but after first separating saccades as being directed towards either the upper visual field (light blue) or lower visual field (light red). The first two rows show visuallyguided saccades (immediate for the first row, and delayed based on a task instruction in the second row; Methods). The third row shows memory-guided saccades towards a blank region of the display. The insets in the first row show saccadic reaction time data (as in Fig. 3B, left) for the same saccades as in the main sequence plots, to highlight the strong presence of a visual field effect on reaction times and a concomitant absence of a visual field effect on saccade kinematics. In all saccade contexts (across rows), the visual field location of the saccade endpoint had minimal effect on saccade kinematics (despite a large effect on saccadic reaction times and despite an asymmetry in SC motor bursts; Fig. 1). The insets in the first row directly replicate the plots in Fig. 4A, C of (Hafed \& Goffart, 2020) for easier comparison of effect sizes for reaction times and kinematics. Error bars denote s.e.m. Note that monkey $\mathrm{N}$ showed a small reduction of peak eye speed for downward saccades when compared to upward saccades only in the memory-guided condition (bottom row), but this effect is opposite from what would be expected if SC motor bursts (Fig. 1) dictated kinematics. The insets were replotted with permission from (Hafed \& Goffart, 2020).

Finally, even though memory-guided saccades were generally slower than visually-guided saccades (compare the bottom row of Fig. 4 to the two rows above it) (Edelman \& Goldberg, 2001; Gnadt \& Andersen, 1988; Hikosaka \& Wurtz, 1983; Powers, Basso, \& Evinger, 2013; Smit, Van Gisbergen, \& Cools, 1987; Stanford \& Sparks, 1994; White, Sparks, \& Stanford, 1994), the above-mentioned kinematic similarity between movements towards the upper 
and lower visual fields still persisted in the memory-guided saccade task. Only in monkey $\mathrm{N}$ (left column of the bottom row of Fig. 4) was there a reduction in downward saccade peak eye speed when compared to upward saccade peak eye speed. However, even in this case, such a reduction was inconsistent with the stronger saccade-related motor bursts for lower visual field saccades seen in the SC neural analyses (Fig. 1). If anything, stronger lower visual

380 field SC motor bursts (along with larger response fields; (Hafed \& Chen, 2016) might predict higher, rather than lower, peak speeds for downward saccades.

Therefore, across a large range of movement sizes and directions, I found minimal kinematic differences between upper and lower visual field saccades, even though other aspects of saccade generation (such as reaction times; insets in Fig. 4) were strongly different, and even though SC saccade-related motor bursts were also different (Fig. 1). reached the same conclusions as for the case of saccade peak radial eye speeds (Fig. 4). The saccade duration versus amplitude curves strongly overlapped for saccades towards the upper (light blue) and lower (light red) visual fields (Fig. 5), and this was true across task contexts. Note how monkey $\mathrm{N}$ compensated for the slightly slower downward memoryguided saccade peak speeds (when compared to upward memory-guided saccade peak speaks) with mildly longer durations for these movements (left column of the bottom row of Fig. 5). This might suggest that there was lower drive for generating this monkey's downward memory-guided saccades in general, which was then compensated for by increased movement durations. Nonetheless, as stated above, this is an opposite effect from what might be expected from the neural burst strengths in the SC (Fig. 1). 
Monkey $\mathrm{N}$
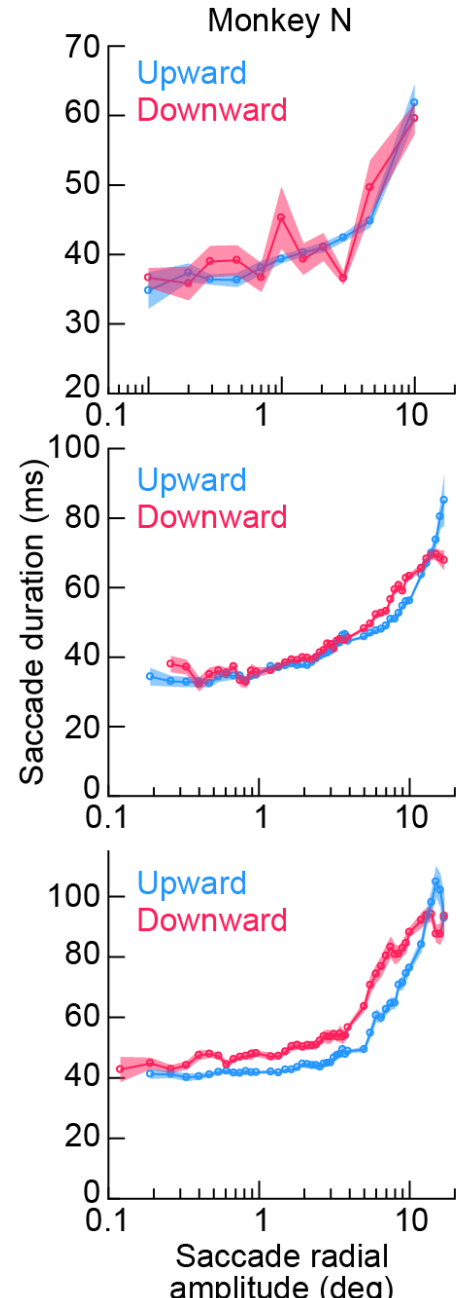

Monkey M
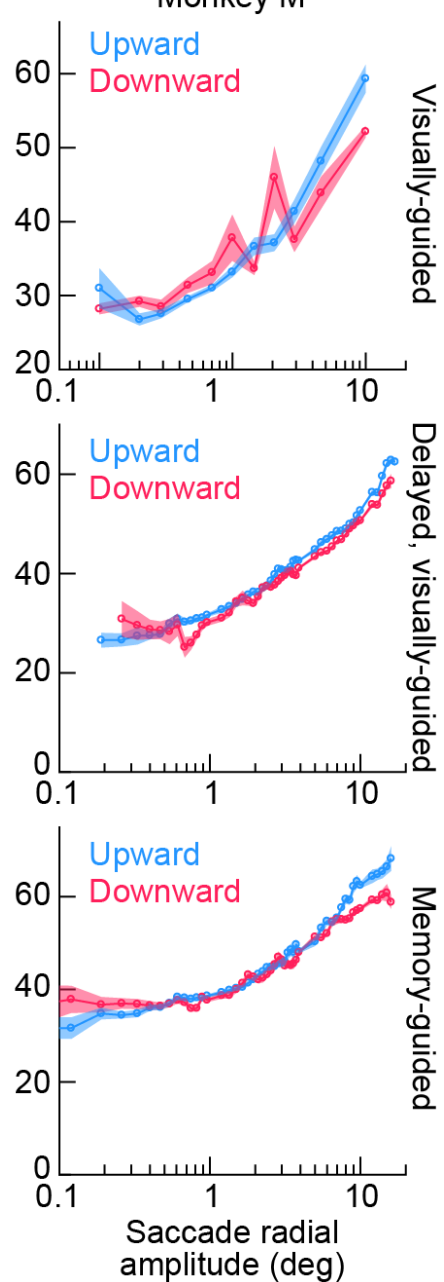

Figure $\mathbf{5}$ Saccade durations as a function of saccade amplitude are also largely insensitive to whether saccades are directed towards the upper or lower visual field. Same as Fig. 4 but now plotting saccade duration as a function of saccade amplitude. Similar conclusions were reached concerning the minimal influence of upper versus lower visual field saccade target locations on saccade kinematics, despite asymmetries in SC motor bursts (Fig. 1). For monkey $\mathrm{N}$ in the memory-guided condition (left panel of the bottom row), the slower lower visual field saccade peak speeds (Fig. 4) meant slightly longer saccade durations when compared to upper visual field saccades (a speed-duration tradeoff). Error bars denote s.e.m.

\section{Discussion}

I described a dissociation between SC saccade-related motor burst strength and movement kinematics. In particular, I confirmed an asymmetry in motor burst strength between upper and lower visual field saccade target locations (Fig. 1). I then found that the kinematics of saccades towards upper and lower visual field locations were not different from each other across a range of movement sizes, directions, and behavioral contexts (Figs. 2-5).

Other examples of dissociations between burst strengths and movement properties are consistent with my interpretation that the SC temporal code does not necessarily dictate moment-to-moment movement kinematics, as might be suggested by some recent models (Goossens \& van Opstal, 2012; Smalianchuk et al., 2018). For example, when making saccades towards a blank (as opposed towards an explicit visual target), a significant fraction 
of SC neurons becomes completely silent at the time of movement onset, even though the movement kinematics are minimally altered (Edelman \& Goldberg, 2001; Mohler \& Wurtz, 1976; Willeke et al., 2019). In this example of saccades towards a blank, it may be argued that the number of neurons that become silent might be extremely small, as was seen in some early studies (Edelman \& Goldberg, 2001). However, in our more recent characterization of this phenomenon with a larger neural database (Willeke et al., 2019), we noticed a much larger population of neurons that became silent. This, in addition to neurons that exhibit altered response field profiles when making saccades towards a blank (Stanford \& Sparks, 1994), suggests a significantly modified SC representation for these movements. Nonetheless, the movements only exhibit mild kinematic alterations (Edelman \& Goldberg, 2001). Therefore, the relationship between movement kinematics and SC motor bursts is relatively loose when making saccades towards a blank, and it was also relatively loose in my analyses of upper and lower visual field target locations.

Another example of a dissociation between saccade motor burst strength and movement kinematics was observed when saccades were driven by combinations of visual and auditory sensory signals, as opposed to only visual signals (Frens \& Van Opstal, 1998). Interestingly, it was again the case in this example that a sensory scenario was relevant and critical for revealing a potential separation between the SC temporal code and movement kinematics. That is, in both the example above of saccades towards a blank as well as the current example of multi-sensory target specification, it was a modification of a sensory property of saccade targets that has allowed observing a dissociation between motor burst strengths and eye movement properties. In the current manuscript's context as well, I was originally motivated by the fact that it was visual sensitivity that was strongly variable between upper and lower visual field locations (Hafed \& Chen, 2016). Indeed, given that stronger visual responses occur in the upper visual field whereas stronger motor responses occur in the lower visual field, it is intriguing to consider the possibility that there might be a general anticorrelation property between visual sensitivity and saccade-related motor burst strength in the SC, for example, in the ubiquitous visual-motor neurons of this structure. burst strength and executed saccade properties (Peel et al., 2020). Specifically, these authors causally perturbed top-down inputs towards the SC through reversible cooling of the cortex, and they found reduced SC burst strengths for metrically similar executed saccades. This study, along with (Frens \& Van Opstal, 1998), (Willeke et al., 2019), (Edelman \& Goldberg, 2001), and (Mohler \& Wurtz, 1976), all showed that for the very same saccade vector (i.e. only within the upper visual field SC representation or only within the lower visual field SC representation), sensory (Edelman \& Goldberg, 2001; Frens \& Van Opstal, 1998; Mohler \& Wurtz, 1976; Willeke et al., 2019) or physiological (Peel et al., 2020) manipulations can indeed alter SC burst strength without strongly altering saccades. Therefore, whether one considers a single saccade vector like in these studies or a comparison of upper versus lower visual field saccades like in my study, a dissociation between SC saccade-related motor bursts and saccade execution still exists. This is important to explicitly point out here, especially because one potential criticism of my current results could be that the existing models of the temporal code dictating saccade kinematics (Goossens \& van Opstal, 2012; Smalianchuk et al., 2018) have primarily focused on a single saccade vector, whereas I compared upward and downward saccades. According to this potential counter argument to my results, by comparing upper and lower visual field saccades, I might have been 
comparing saccades in which structures downstream from the SC would compensate for the SC asymmetry shown in Fig. 1 and in (Hafed \& Chen, 2016). Because the above-mentioned studies all showed dissociations between the SC temporal code and saccade properties for the very same saccade vector, just like the models of the SC temporal code (Goossens \& van Opstal, 2012; Smalianchuk et al., 2018), this counter argument does not seem to be too strong. In addition, this potential counter argument might imply that the large SC asymmetry in both saccade-related movement burst strengths (Fig. 1) and movement response field sizes (Hafed \& Chen, 2016) just becomes nulled downstream of the SC, which creates the problem of why the asymmetry exists in the $\mathrm{SC}$ at all in the first place. It also ignores the fact that the asymmetry has real consequences for saccade latencies, saccade accuracy, and the likelihood of express saccades (Hafed \& Chen, 2016). Rather, I believe that the SC asymmetry motivates investigating what the functional role of SC motor bursts is, and in a more general framework than just one of controlling saccade kinematics.

My current results also provide complementary evidence to a phenomenon that we recently studied (Buonocore et al., 2021). In that most recent study, we altered saccade kinematics by strategically injecting "visual" bursts into the SC at spatial sites beyond the vector endpoints of the currently executed movements (Buonocore et al., 2021). We found alterations in movement kinematics (Buonocore et al., 2017), which were lawfully related to the amounts of visual spikes that we injected onto the SC map around the time of movement triggering (Buonocore et al., 2021). However, critically, such alterations occurred in the absence of strong alterations in the motor bursts themselves (for the neurons generating the originally planned saccades). This was surprising for a variety of reasons, including ideas related to lateral connectivity patterns in the SC, as we recently discussed (Buonocore et al., 2021). However, it also represented an opportunity for me to explicitly ask, in the current study, whether or not the temporal code was indeed as tightly linked to individual movement kinematics, as suggested by some recent models. Therefore, here, I took the opposite approach from our recent study: I identified a situation in which the motor bursts were different from each other for two different sets of saccades, and I showed that the saccade kinematics in the two groups of movements were the same. Therefore, either with altered movements and minimally-altered movement commands (Buonocore et al., 2021), or with minimally-altered movements and significantly altered movement commands (this work), there is a dissociation between saccade kinematics and SC motor burst strengths. This is also consistent with evidence that saccades can have similar metrics even after altering SC activity through reversible inactivation of the frontal eye fields (Peel et al., 2020).

Recent work has suggested that the SC can support high level perceptual and cognitive phenomena (Basso \& May, 2017; Krauzlis, Lovejoy, \& Zenon, 2013). For example, the SC causally influences selective behaviors (Lovejoy \& Krauzlis, 2010; Zenon \& Krauzlis, 2012), and it even shapes object-related visual representations in the ventral visual processing stream (Bogadhi, Katz, Bollimunta, Leopold, \& Krauzlis, 2021). This is in addition to established roles for the SC in target selection (Horwitz \& Newsome, 1999; Krauzlis, Liston, \& Carello, 2004; McPeek \& Keller, 2004). All of this evidence suggests the SC might occupy a functional level that is slightly more abstract than that of specifying individual millisecondby-millisecond movement evolution, consistent with my results. Thus, it might suffice for the SC to specify movement metrics, via the spatial code, and also potentially contribute to the decision of when to trigger an eye movement, as recently suggested (Jagadisan \& Gandhi, 
bioRxiv preprint doi: https://doi.org/10.1101/2021.06.24.449726; this version posted September 10, 2021. The copyright holder for this

preprint (which was not certified by peer review) is the author/funder, who has granted bioRxiv a license to display the preprint in perpetuity. It is made available under aCC-BY 4.0 International license.

523 2019). The rest can be handled by downstream oculomotor control structures. If this is

524 indeed the case, then a critical and urgent question for research in the immediate future is:

525 what is, ultimately, the functional role of the SC temporal code in visual-motor behavior and

526 perception? 


\section{Acknowledgements}

I was funded by the Deutsche Forschungsgemeinschaft (DFG) through Research Unit: FOR1847 (project: A6: HA6749/2-1).

\section{Declaration of interests}

The author declares no competing interests.

\section{Methods}

I analyzed data from two previously published studies focusing on other topics. In the first, neural activity from the SC and saccadic behavior were recorded from two adult, male rhesus macaque monkeys ( $P$ and N) (Hafed \& Chen, 2016). I analyzed both neural activity and behavior from that study (referred to here as dataset 1 ). In the second study, saccadic behavior was recorded from monkey N and a third monkey (M) (Hafed \& Goffart, 2020); I analyzed additional behavioral parameters from that study (referred to here as dataset 2). As described in both previous publications, the animal experiments were approved by the Regierungspräsidium Tübingen, under license $\mathrm{CIN} 3 / 13$, and they were in accordance with the German and European directives on the use of animals in research.

In what follows, I describe methods relevant for the current work.

\section{Animal preparation}

For SC recording, a recording chamber was implanted centered on the midline in both monkeys. The midline positioning of the chamber allowed recording from both the right and left SC in each animal. Magnetic resonance images (MRI's) obtained prior to the experiments aided in chamber implant alignment. We aimed for quasi-orthogonal electrode penetrations (relative to the SC curvature) at eccentricities we typically use in experiments (e.g. 5-15 deg).

Before receiving the chamber implants, the animals were also implanted with head-holding apparatuses and scleral search coils for eye tracking, as described earlier (Chen \& Hafed, 2013; Chen, Ignashchenkova, Thier, \& Hafed, 2015; Hafed \& Ignashchenkova, 2013). The scleral search coils allowed using the magnetic induction technique for measuring eye positions (Fuchs \& Robinson, 1966; Judge, Richmond, \& Chu, 1980). Specifically, a coil of wire was implanted around the sclera of the eye and below the conjunctiva. The animals were then seated near the middle of a cube in which alternating magnetic fields induced electrical current (which depended on ocular position) in the implanted scleral coil; we measured and calibrated this electrical current. 
For both neural and behavioral analyses, the monkeys performed classic saccade generation tasks. In the immediate, visually-guided saccade task, the monkey first fixated a central spot. After a variable delay, the spot was jumped to a new location, and a saccade to follow the spot was triggered. In the delayed version of the same task, during initial fixation, the fixation spot remained visible while an eccentric spot was presented. The monkey was required to maintain gaze fixation and withhold any reflexive orienting towards the eccentric spot for as long as the central fixation spot was visible. After the fixation spot was removed, the monkey generated a saccade towards the (still visible) eccentric spot. Finally, in the memory-guided saccade task, during initial fixation, the eccentric spot was only flashed briefly (for approximately $50 \mathrm{~ms}$ ). A delay period then ensued in which only the fixation spot was visible, and the monkey was required to maintain gaze fixation on it. At the end of this so-called memory period, the fixation spot was extinguished, instructing the monkey to generate a saccade towards the remembered location of the previous flash (i.e. towards a blank location on the display).

The delayed, visually-guided saccade task was used for all neural analyses reported in this study (dataset 1). For behavioral analyses, I used the delayed saccade task in the same dataset (e.g. Fig. 2), as well as all 3 saccade tasks from dataset 2. I also checked neural responses for the memory-guided saccade task in dataset 1 , and the asymmetry of SC motor bursts reported in Fig. 1 was still present; however, I do not show these results for brevity.

In all cases, stimuli were presented on cathode ray tube (CRT) displays, with stimulus luminances and dimensions having been described earlier (Hafed \& Chen, 2016; Hafed \& Goffart, 2020). The timing of trial events in the tasks was also described earlier. For the present study, the primary focus was on the individual saccade kinematics at the ends of all trials, irrespective of timing parameters, such as the length of the delay or memory period, and irrespective of the exact stimulus visual properties. The effects of these factors (such as trial timing or visual stimulus properties) were described earlier (Chen \& Hafed, 2018; Chen, Sonnenberg, Weller, Witschel, \& Hafed, 2018; Hafed \& Chen, 2016; Hafed \& Goffart, 2020).

\section{Neural data analyses}

I analyzed peri-saccadic firing rates, as we did previously (Hafed \& Chen, 2016). For each neuron in the database of the previous study (containing $>400$ neurons), we had identified (for saccade-related neurons) the saccades towards the neuron's preferred movementrelated response field location (i.e. the locations for which the neuron's saccade-related bursts were the strongest). In the present study, I analyzed the firing rates for these preferred saccades. However, I constrained the choice of neurons according to the needs of the current study. Specifically, besides only considering neurons with saccade-related bursts, I matched neural depths between neurons from the upper and lower visual field representations of the SC (e.g. Fig. 1A). Specifically, since saccade-related motor bursts in the SC can vary in strength as a function of depth of the neurons from the SC surface (Massot et al., 2019), I only compared motor bursts after selecting neurons from the upper and lower visual field representations that had matched depths. 
To do so, I first considered all neurons in the upper and lower visual field representations having a depth of 600-1850 $\mu \mathrm{m}$ from the SC surface. This range of depths is consistent with known depths of saccade-related activity in the SC (Massot et al., 2019). Importantly, for the present purposes, this range of depths contained clear overlap between neurons in the upper and lower visual field SC representations (Fig. 1A). This allowed comparing the strengths of motor bursts between the selected depth-matched neurons. The resulting neural database had 167 neurons (Fig. 1).

To further confirm that there was no confound of neural depth from the SC surface in interpreting a visual field asymmetry in motor burst strength, I was concerned that the curvature of the SC surface could introduce systematic biases in depths of upper versus lower visual field neurons from the SC surface. For example, it could potentially be the case that the 3-dimensional SC surface curvature combined with a constant electrode approach angle dictated by the recording chamber might systematically skew depth estimates: medial (upper visual field) electrode locations might potentially have depth estimates that could be systematically different from lateral (lower visual field) electrode locations in the chamber. This could simply be a function of whether or not a given electrode track was more or less perpendicular to the local SC surface topography at a given site. In my second analysis of neural activity, I therefore picked a range of electrode locations in which I expected minimal changes in SC curvature between upper and lower visual field representations. For example, mapping the SC surface topography on the anatomical SC (Chen et al., 2019) might suggest a similar relationship between electrode angle and SC surface for upper and lower visual field representations near the horizontal meridian and within a specific range of movement amplitudes. I therefore specifically picked neurons with movement-related response field hotspots near the horizontal meridian (within 30 deg direction in either the upper or lower visual fields) and with radial eccentricities of only 5-15 deg. I also picked a narrower depth of neurons for the comparison (1100-1900 $\mu \mathrm{m}$ from SC surface). With this more strict neural database (31 neurons), I again plotted peri-saccadic firing rates for neurons in the upper and lower visual field representations (Fig. 1C).

In all cases, a neuron was considered to be part of the upper or lower visual field representation if its preferred saccade (i.e. the movement-related response field hotspot location) was in the upper or lower visual field, respectively. This was also consistent with the known SC topographic representation (Chen et al., 2019; Cynader \& Berman, 1972; Hafed \& Chen, 2016; Robinson, 1972), and it was already done in our previous study (Hafed \& Chen, 2016).

To statistically compare saccade-related activity strength between the upper and lower visual field representations in the SC, I measured the average firing rate in the final $50 \mathrm{~ms}$ before saccade onset for each neuron. I then statistically compared the firing rates of all neurons having movement-related response field hotspot locations in the upper visual field to the firing rates of all neurons having response field hotspot locations in the lower visual 


\section{Behavioral data analyses}

All saccades were detected for the previous two studies (Hafed \& Chen, 2016; Hafed \& Goffart, 2020). Here, I analyzed the kinematic properties of the movements.

For dataset 1 , I picked saccades having +45 or -45 deg direction from the horizontal meridian (i.e. oblique saccades). I then picked 5 radial amplitude categories to characterize 5 different ranges of saccade sizes (Fig. 2). The categories were: 3, 5, 7, 10, and 13 deg. For each of these categories, I picked all saccades landing within a radius of $0.5,0.8,1,2$, and 3 deg from the designated amplitude/direction category, respectively. For example, for saccades of 7 deg amplitude and +45 deg direction, I picked all saccades that were upward and oblique, and that were directed towards an eccentricity of $7 \mathrm{deg}$, and that landed within a radius of 1 deg from this eccentricity. Similarly, for 3 deg saccades of +45 deg direction, I picked all upward oblique movements towards an eccentricity of $3 \mathrm{deg}$ and landing within a radius of 0.5 deg from it. This meant that I had amplitude- and direction-matched saccades for either the oblique upward or the oblique downward movements. I then plotted the trajectories (Fig. 2A) and radial speed profiles (Fig. 2B) of all of these saccades. Since the speeds of temporally-directed saccades could be different from the speeds of nasally-directed saccades for a given tracked eye, I analyzed rightward and leftward saccades separately in this dataset (Fig. 2B). However, in dataset 2, all saccade directions were combined, and with similar conclusions.

For dataset 2, I had a large range of saccade amplitudes and directions to analyze (Hafed \& Goffart, 2020). I plotted the main sequence relationship (Bahill et al., 1975; Zuber et al., 1965) for these saccades after separating them into two groups: saccades towards the upper visual field and saccades towards the lower visual field. I plotted both the main sequence relationship of peak speed versus movement amplitude (Fig. 4) and saccade duration versus movement amplitude (Fig. 5). For comparison, I included a plot of saccadic reaction times for the same saccades in Fig. 4. This was a replotting of the reaction time data already reported earlier (Hafed \& Goffart, 2020), and I included it here for easier comparison of the difference in effects of visual field location on saccade kinematics and saccade reaction times. In total, I analyzed 1246, 928 visually-guided saccade trials, 6147, 5871 delayed, visually-guided saccade trials, and 6428, 9631 memory-guided saccade trials from monkeys $N$ and $M$, respectively. The numbers of trials for the behavioral analyses from dataset 1 are reported in the figure legend of Fig. 2. 
Bahill, A. T., Clark, M. R., \& Stark, L. (1975). The main sequence, a tool for studying human eye movements. Mathematical Biosciences, 24(3-4), 191-204. Colliculus. Annu Rev Vis Sci. doi:10.1146/annurev-vision-102016-061234

719

Bogadhi, A. R., Katz, L. N., Bollimunta, A., Leopold, D. A., \& Krauzlis, R. J. (2021). Midbrain activity shapes high-level visual properties in the primate temporal cortex. Neuron, 109(4), 690-699 e695. doi:10.1016/j.neuron.2020.11.023

Buonocore, A., Chen, C. Y., Tian, X., Idrees, S., Munch, T. A., \& Hafed, Z. M. (2017). Alteration of the microsaccadic velocity-amplitude main sequence relationship after visual transients: implications for models of saccade control. J Neurophysiol, 117(5), 18941910. doi:10.1152/jn.00811.2016

Buonocore, A., McIntosh, R. D., \& Melcher, D. (2016). Beyond the point of no return: effects of visual distractors on saccade amplitude and velocity. J Neurophysiol, 115(2), 752762. doi:10.1152/jn.00939.2015

Buonocore, A., Tian, X., Khademi, F., \& Hafed, Z. M. (2021). Instantaneous movementunrelated midbrain activity modifies ongoing eye movements. Elife, 10. doi:10.7554/eLife.64150

Chen, C. Y., \& Hafed, Z. M. (2013). Postmicrosaccadic enhancement of slow eye movements. The Journal of neuroscience : the official journal of the Society for Neuroscience, 33(12), 5375-5386. doi:10.1523/JNEUROSCI.3703-12.2013

Chen, C. Y., \& Hafed, Z. M. (2018). Orientation and Contrast Tuning Properties and Temporal Flicker Fusion Characteristics of Primate Superior Colliculus Neurons. Front Neural Circuits, 12, 58. doi:10.3389/fncir.2018.00058

Chen, C. Y., Hoffmann, K. P., Distler, C., \& Hafed, Z. M. (2019). The Foveal Visual Representation of the Primate Superior Colliculus. Curr Biol, 29(13), 2109-2119 e2107. doi:10.1016/j.cub.2019.05.040

Chen, C. Y., Ignashchenkova, A., Thier, P., \& Hafed, Z. M. (2015). Neuronal Response Gain Enhancement prior to Microsaccades. Curr Biol, 25(16), 2065-2074. doi:10.1016/j.cub.2015.06.022

Chen, C. Y., Sonnenberg, L., Weller, S., Witschel, T., \& Hafed, Z. M. (2018). Spatial frequency sensitivity in macaque midbrain. Nature communications, 9(1), 2852. doi:10.1038/s41467-018-05302-5 
Cynader, M., \& Berman, N. (1972). Receptive-field organization of monkey superior colliculus. J Neurophysiol, 35(2), 187-201.

Edelman, J. A., \& Goldberg, M. E. (2001). Dependence of saccade-related activity in the primate superior colliculus on visual target presence. J Neurophysiol, 86(2), 676-691. doi:10.1152/jn.2001.86.2.676

Frens, M. A., \& Van Opstal, A. J. (1998). Visual-auditory interactions modulate saccaderelated activity in monkey superior colliculus. Brain Res Bull, 46(3), 211-224. doi:S0361-9230(98)00007-0 [pii]

Fuchs, A. F., \& Robinson, D. A. (1966). A method for measuring horizontal and vertical eye movement chronically in the monkey. Journal of applied physiology, 21(3), 10681070.

Gnadt, J. W., \& Andersen, R. A. (1988). Memory related motor planning activity in posterior parietal cortex of macaque. Exp Brain Res, 70(1), 216-220. doi:10.1007/BF00271862

Goossens, H. H., \& Van Opstal, A. J. (2000). Blink-perturbed saccades in monkey. II. Superior colliculus activity. J Neurophysiol, 83(6), 3430-3452.

Goossens, H. H., \& van Opstal, A. J. (2012). Optimal control of saccades by spatial-temporal activity patterns in the monkey superior colliculus. PLoS computational biology, 8(5), e1002508. doi:10.1371/journal.pcbi.1002508

Greene, H. H., Brown, J. M., \& Dauphin, B. (2014). When do you look where you look? A visual field asymmetry. Vision Res, 102, 33-40. doi:10.1016/j.visres.2014.07.012

Greenwood, J. A., Szinte, M., Sayim, B., \& Cavanagh, P. (2017). Variations in crowding, saccadic precision, and spatial localization reveal the shared topology of spatial vision. Proc Natl Acad Sci U S A, E3573-E3582. doi:www.pnas.org/cgi/doi/10.1073/pnas.1615504114

Hafed, Z. M., \& Chen, C. Y. (2016). Sharper, Stronger, Faster Upper Visual Field Representation in Primate Superior Colliculus. Curr Biol, 26(13), 1647-1658. doi:10.1016/j.cub.2016.04.059

Hafed, Z. M., \& Goffart, L. (2020). Gaze direction as equilibrium: more evidence from spatial and temporal aspects of small-saccade triggering in the rhesus macaque monkey. $J$ Neurophysiol, 123(1), 308-322. doi:10.1152/jn.00588.2019

Hafed, Z. M., \& Ignashchenkova, A. (2013). On the dissociation between microsaccade rate and direction after peripheral cues: microsaccadic inhibition revisited. J Neurosci, 33(41), 16220-16235. doi:10.1523/JNEUROSCI.2240-13.2013 
Hikosaka, O., \& Wurtz, R. H. (1983). Visual and oculomotor functions of monkey substantia nigra pars reticulata. III. Memory-contingent visual and saccade responses. J Neurophysiol, 49(5), 1268-1284.

Horwitz, G. D., \& Newsome, W. T. (1999). Separate signals for target selection and movement specification in the superior colliculus. Science, 284(5417), 1158-1161. doi:10.1126/science.284.5417.1158

Jagadisan, U. K., \& Gandhi, N. J. (2019). Population temporal structure supplements the rate code during sensorimotor transformations. bioRxiv. doi:10.1101/132514

Judge, S. J., Richmond, B. J., \& Chu, F. C. (1980). Implantation of magnetic search coils for measurement of eye position: an improved method. Vision Res, 20(6), 535-538.

Katnani, H. A., \& Gandhi, N. J. (2012). The relative impact of microstimulation parameters on movement generation. J Neurophysiol, 108(2), 528-538. doi:10.1152/jn.00257.2012

Krauzlis, R. J., Liston, D., \& Carello, C. D. (2004). Target selection and the superior colliculus: goals, choices and hypotheses. Vision Res, 44(12), 1445-1451. doi:10.1016/j.visres.2004.01.005

S0042698904000264 [pii]

Krauzlis, R. J., Lovejoy, L. P., \& Zenon, A. (2013). Superior colliculus and visual spatial attention. Annu Rev Neurosci, 36, 165-182. doi:10.1146/annurev-neuro-062012170249

Lee, C., Rohrer, W. H., \& Sparks, D. L. (1988). Population coding of saccadic eye movements by neurons in the superior colliculus. Nature, 332(6162), 357-360. doi:10.1038/332357a0

Lovejoy, L. P., \& Krauzlis, R. J. (2010). Inactivation of primate superior colliculus impairs covert selection of signals for perceptual judgments. Nat Neurosci, 13(2), 261-266. doi:nn.2470 [pii]

$10.1038 / \mathrm{nn} .2470$

Massot, C., Jagadisan, U. K., \& Gandhi, N. J. (2019). Sensorimotor transformation elicits systematic patterns of activity along the dorsoventral extent of the superior colliculus in the macaque monkey. Commun Biol, 2, 287. doi:10.1038/s42003-019-0527-y

McPeek, R. M., \& Keller, E. L. (2004). Deficits in saccade target selection after inactivation of superior colliculus. Nat Neurosci, 7(7), 757-763. doi:10.1038/nn1269

nn1269 [pii]

Mohler, C. W., \& Wurtz, R. H. (1976). Organization of monkey superior colliculus: intermediate layer cells discharging before eye movements. J Neurophysiol, 39(4), 722-744. 
Munoz, D. P., \& Wurtz, R. H. (1995a). Saccade-related activity in monkey superior colliculus. I. Characteristics of burst and buildup cells. J Neurophysiol, 73(6), 2313-2333.

Munoz, D. P., \& Wurtz, R. H. (1995b). Saccade-related activity in monkey superior colliculus. II. Spread of activity during saccades. J Neurophysiol, 73(6), 2334-2348.

Peel, T. R., Dash, S., Lomber, S. G., \& Corneil, B. D. (2020). Frontal eye field inactivation alters the readout of superior colliculus activity for saccade generation in a task-dependent manner. J Comput Neurosci. doi:10.1007/s10827-020-00760-7

Powers, A. S., Basso, M. A., \& Evinger, C. (2013). Blinks slow memory-guided saccades. J Neurophysiol, 109(3), 734-741. doi:10.1152/jn.00746.2012

Robinson, D. A. (1972). Eye movements evoked by collicular stimulation in the alert monkey. Vision Res, 12(11), 1795-1808. doi:0042-6989(72)90070-3 [pii]

Schiller, P. H., \& Koerner, F. (1971). Discharge characteristics of single units in superior colliculus of the alert rhesus monkey. J Neurophysiol, 34(5), 920-936.

Schlykowa, L., Hoffmann, K. P., Bremmer, F., Thiele, A., \& Ehrenstein, W. H. (1996). Monkey saccadic latency and pursuit velocity show a preference for upward directions of target motion. Neuroreport, 7(2), 409-412.

Smalianchuk, I., Jagadisan, U. K., \& Gandhi, N. J. (2018). Instantaneous Midbrain Control of Saccade Velocity. J Neurosci, 38(47), 10156-10167. doi:10.1523/JNEUROSCI.096218.2018

Smit, A. C., Van Gisbergen, J. A., \& Cools, A. R. (1987). A parametric analysis of human saccades in different experimental paradigms. Vision Res, 27(10), 1745-1762. doi:10.1016/0042-6989(87)90104-0

Sommer, M. A., \& Wurtz, R. H. (2004). What the brain stem tells the frontal cortex. I. Oculomotor signals sent from superior colliculus to frontal eye field via mediodorsal thalamus. J Neurophysiol, 91(3), 1381-1402. doi:10.1152/jn.00738.2003

00738.2003 [pii]

Sparks, D. L., Holland, R., \& Guthrie, B. L. (1976). Size and distribution of movement fields in the monkey superior colliculus. Brain Res, 113(1), 21-34. doi:10.1016/00068993(76)90003-2

Stanford, T. R., \& Sparks, D. L. (1994). Systematic errors for saccades to remembered targets: evidence for a dissociation between saccade metrics and activity in the superior colliculus. Vision Res, 34(1), 93-106. 
van Opstal, A. J., \& Goossens, H. H. (2008). Linear ensemble-coding in midbrain superior colliculus specifies the saccade kinematics. Biol Cybern, 98(6), 561-577. doi:10.1007/s00422-008-0219-z

Waitzman, D. M., Ma, T. P., Optican, L. M., \& Wurtz, R. H. (1988). Superior colliculus neurons provide the saccadic motor error signal. Exp Brain Res, 72(3), 649-652.

Waitzman, D. M., Ma, T. P., Optican, L. M., \& Wurtz, R. H. (1991). Superior colliculus neurons mediate the dynamic characteristics of saccades. J Neurophysiol, 66(5), 1716-1737.

White, J. M., Sparks, D. L., \& Stanford, T. R. (1994). Saccades to remembered target locations: an analysis of systematic and variable errors. Vision Res, 34(1), 79-92. doi:10.1016/0042-6989(94)90259-3

Willeke, K. F., Tian, X., Buonocore, A., Bellet, J., Ramirez-Cardenas, A., \& Hafed, Z. M. (2019). Memory-guided microsaccades. Nature communications, 10(1), 3710. doi:10.1038/s41467-019-11711-x

Wurtz, R. H., \& Goldberg, M. E. (1971). Superior colliculus cell responses related to eye movements in awake monkeys. Science, 171(966), 82-84.

Zenon, Z., \& Krauzlis, R. J. (2012). Attention deficits without cortical neuronal deficits. Nature, 489, 434-437.

Zhou, W., \& King, W. M. (2002). Attentional sensitivity and asymmetries of vertical saccade generation in monkey. Vision Res, 42(6), 771-779.

Zuber, B. L., Stark, L., \& Cook, G. (1965). Microsaccades and the velocity-amplitude relationship for saccadic eye movements. Science, 150(702), 1459-1460. 